\title{
DISEÑO, CONSTRUCCIÓN Y MODELAMIENTO DE UN REACTOR TUBULAR DE LABORATORIO PARA REALIZAR HIDRÓLISIS Y SAPONIFICACIÓN
}

Responsable: Ing. Juan Viloche Bazán

\section{RESUMEN}

Primero se determinó el orden de reacción de hidrólisis de cristal violeta que fue una seudoreacción de primer orden y un modelo de segundo orden para la saponificación del acetato de etilo, que permitió calcular el volumen del reactor para cada reacción. El reactor tubular (PFR) se construyó de polietileno de $12 \mathrm{~m}$ de longitud, un diámetro interno de $0,95 \mathrm{~cm}$ y una capacidad de $850 \mathrm{~cm}^{3}$

El reactorPFR está enrollado helicoidalmente a lo largo de 30 $\mathrm{cm}$ en un soporte de acrílico cristal de $5 \mathrm{~mm}$. de espesor y $15 \mathrm{~cm}$ de diámetro externo y sumergido en agua a temperatura controlada que permita modificar la temperatura del sistema. La conversión de diseño es aproximadamente del $80 \%$, tanto para la hidrólisis del cristal violeta como para la saponificación del acetato de etilo.

\section{INTRODUCCIÓN}

Uno de los problemas fundamentales que se le presentan al ingeniero químico que desea diseñar o simular la operación de un reactor es el conocimiento de los modelos cinéticos. El modelo cinético tiene que ser determinado experimentalmente 0 se puede obtener mediante una búsqueda bibliográfica.

La modelación y simulación de un reactor es de invaluable ayuda para determinar en qué dirección se modificarán las variables de operación y de proceso para maximizar la producción a los menores costos, esta modelación requiere de un entendimiento claro de fenómenos cinéticos, de superficie y de transporte.

\section{REVISIÓN BIBLIOGRÁFICA}

Las reacciones con las cuales se va a trabajar en el presente proyecto son reacciones de primer orden y reacciones de segundo orden.

\section{1) Reacciones de Primer Orden}

$$
-\mathrm{r}_{\mathrm{A}}=-\frac{\mathrm{dC}_{\mathrm{A}}}{\mathrm{dt}}=\mathrm{kC}_{\mathrm{A}}
$$

Que luego de ser integrada tiene la forma

$$
\operatorname{Ln}\left(\frac{C_{A_{0}}}{C_{A}}\right)=-\operatorname{Ln}\left(1-X_{A}\right)=k t
$$

\section{2) Reacciones de Segundo Orden}

Son la mayoria de las reacciones bimoleculares que se realiza en la naturaleza y tiene la forma siguiente:

$$
\begin{array}{r}
\mathrm{aA}+\mathrm{bB} \longrightarrow \text { Productos } \\
-\mathrm{r}_{\mathrm{A}}=-\frac{\mathrm{dC}_{\mathrm{A}}}{\mathrm{dt}}=\mathrm{kC}_{\mathrm{A}} \mathrm{C}_{\mathrm{B}}
\end{array}
$$

Aplicando el grado de conversión se tiene

$$
\frac{\mathrm{dX}_{\mathrm{A}}}{\mathrm{dt}}=\mathrm{k} \cdot \mathrm{C}_{\mathrm{A}_{\mathrm{O}}}\left(1-\mathrm{X}_{\mathrm{A}}\right)\left(\mathrm{M}-\mathrm{X}_{\mathrm{A}}\right)
$$

Como $M=C_{B} / C_{A_{0}}$ se puede integrar la Ecuación anterior obteniendo

$$
\operatorname{Ln}\left(\frac{M-X_{A}}{M\left(1-X_{A}\right)}\right)=\left(C_{B_{0}}-C_{A_{0}}\right) k \cdot t
$$

\section{3) Diseño de un Reactor Tubular.}

$$
\int_{0}^{\mathrm{V}} \mathrm{dV}=\mathrm{F}_{\mathrm{Ao}} \int_{0}^{\mathrm{x}_{\mathrm{A}}} \frac{\mathrm{dX} \mathrm{A}}{-\mathrm{r}_{\mathrm{A}}}
$$

Si sustituimos la ley de velocidad $\left(-r_{A}\right)$ para el caso de una reacción de hidrólisis del Cristal Violeta (reacción de primer orden) y para el caso de Saponificación de Acetato de Etilo ( reacción de segundo orden) se puede obtener el volumen real del reactor. 


\section{MATERIALY MÉTODOS}

\section{1) Reactivos}

- Agua

- Cristal violeta

- Acetato de Etilo $\quad(\rho=0,9025$ ramos $/ \mathrm{ml})$

- $\mathrm{NaOH} \quad(97,7 \%$ pureza)

- $\mathrm{Hcl}$ (37\% pureza)

\section{2) Materiales y Equipos}

- Reactor Batch de 1000 ml de capacidad (vaso de precipitados)

- Termostato

- Interfase de adquisición de datos

- Sensor de conductimetria

- Sensor de temperatura

- Bomba peristáltica

- Reactor tubular construido

- Manguera de polietileno

\section{3) Métodos de Medición de Datos Experimentales Usados}

Hidrólisis de Cristal Violeta:

Primero se debe determinar una curva de patrón y con esta curva patrón determinar en cualquier instante la concentración del Cristal Violeta cuando se está hidrolizando.

\section{Saponificación de Acetato de Etilo:}

La saponificación se puede expresar mediante la siguiente ecuación:

\section{$\mathrm{CH}_{3} \mathrm{COOC}_{2} \mathrm{H}_{5}+\mathrm{NaOH} \rightarrow \mathrm{CH}_{3} \mathrm{COONa}+\mathrm{C}_{2} \mathrm{H}_{5} \mathrm{OH}$}

La conductividad del sistema reaccionante disminuye, debido a que la conductividad equivalente del ión $\mathrm{OH}$ es mayor que la del ión acetato, y éstos sustituyen a los $\mathrm{OH}$ en el transcurso de la reacción (el acetato de etilo y el alcohol etilico no son iónicos).

Como la conductividad es proporcional a la concentración del $\mathrm{OH}$, se puede establecer un modelo que relaciona la conductividad con el grado ce conversión.

$$
X_{A}=\left(\frac{L o-L_{t}}{L o-L \infty}\right)
$$

Donde

$$
\begin{aligned}
& X_{A} \quad=\text { grado de conversión } \\
& \text { Lo = conductividad inicial de } \\
& \text { la solución } \\
& \mathrm{L}_{1} \quad \text { = conductividad } \mathrm{a} \text { un } \\
& \text { determinado tiempo } \\
& \mathrm{L}=\text { = conductividad a un tiempo } \\
& \text { Prolongado }
\end{aligned}
$$

\section{RESULTADOS}

1) Curvas de Calibración y Modelo Cinético

Hidrólisis de Cristal violeta

$\mathrm{CCV}=3,131 \mathrm{E}-07+1,238 \mathrm{E}-05(\mathrm{ABS}) \quad \mathrm{R}^{2}=$ $9,966 \mathrm{E}-01$

$-\mathrm{r}_{\mathrm{CV}}=0,174 \mathrm{C}_{\mathrm{CV}}$

\section{Saponificación de Acetato de Etilo}

El seguimiento de la reacción de saponificación se realizó mediante las medidas de la conductividad a diferentes temperaturas obteniéndose el siguiente resultado:

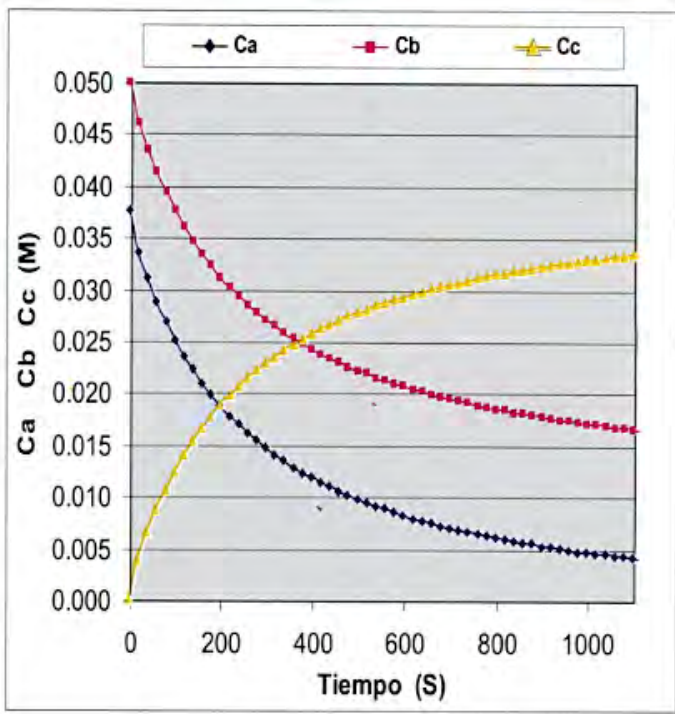

Figura $\mathrm{N}^{\circ}$ 01: Variación de la concentración de reactantes y productos como función del tiempo.

$$
\mathrm{k}=2,082\left(10^{9}\right) \mathrm{e}^{\left(-\frac{5824}{\mathrm{~T}}\right)}
$$

\section{2) Diseño del Reactor PFR para Hidrólisis de Cristal Violeta}

Reemplazando la ecuación (1) en la ecuación (6) se reduce a:

$$
\mathrm{V}=\mathrm{Q} \int_{0}^{\mathrm{X}_{\mathrm{A}}} \frac{\mathrm{dX} \mathrm{A}_{\mathrm{A}}}{\mathrm{k} *\left(1-X_{A}\right)}
$$

Dondek es igual $0,1741 / \mathrm{min}, \mathrm{C}_{\mathrm{A}_{0}}$ es $7,33 \mathrm{E}-06 \mathrm{~mol} / \mathrm{l}$ (concentración inicial de Cristal Violeta), Q es $60 \mathrm{ml} / \mathrm{min}$, $V$ es volumen del reactor tubular $\left(\mathrm{cm}^{3}\right)$ de diámetro $D$ (cm) y altura $\mathrm{H}(\mathrm{cm})$.

Con los datos de conversión obtenidos experimentalmente se construye la curva 


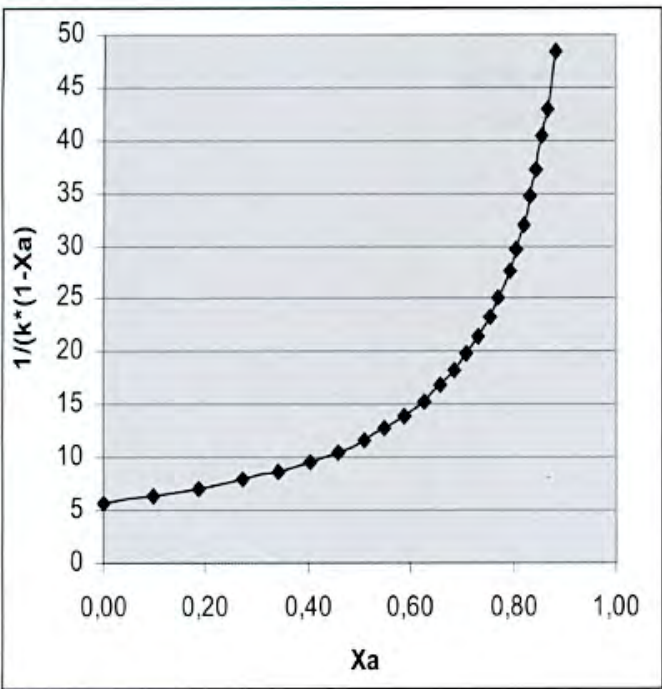

Figura $N^{\circ}$ 02: Cálculo del volumen del reactor PFR para hidrólisis de Cristal Violeta

$V=60 \mathrm{~cm} / \mathrm{min}^{*} 12,2670268=613,35134 \mathrm{~cm}$

Luego el $\mathrm{H}$ es $9,65 \mathrm{~m}$

\section{3) Diseño del Reactor PFR para Saponificación de Acetato de Etilo}

El volumen del reactor PFR se calcula por

$$
\mathrm{V}=\mathrm{Q} \int_{0}^{\mathrm{X}_{\mathrm{A}}} \frac{\mathrm{d} \mathrm{X}_{\mathrm{A}}}{\mathrm{k} \cdot \mathrm{C}_{\mathrm{Ao}}\left(1-X_{A}\right)\left(M-X_{A}\right)}
$$

Se realizan las pruebas experimentales y se procede de igual manera como se procedió para el cálculo del volumen del PFR para la hidrólisis de Cristal Violeta.

\begin{tabular}{|c|c|c|}
\hline $\mathrm{Xa}$ & $\mathrm{Q}(\mathrm{ml} / \mathrm{min})$ & $\mathrm{V}\left(\mathrm{cm}^{3}\right)$ \\
\hline 0,8 & 60 & 704,742395 \\
\hline 0,8 & 50 & 587,285329 \\
\hline 0,8 & 40 & 469,828263 \\
\hline $\mathrm{Xa}$ & $\mathrm{Q}(\mathrm{ml} / \mathrm{min})$ & $\mathrm{V}\left(\mathrm{cm}^{3}\right)$ \\
\hline 0,85 & 60 & 900,21149 \\
\hline 0,85 & 50 & 750,176241 \\
\hline 0,85 & 40 & 600,140993 \\
\hline $\mathrm{Xa}$ & $\mathrm{Q}(\mathrm{ml} / \mathrm{min})$ & $\mathrm{V}\left(\mathrm{cm}^{3}\right)$ \\
\hline 0,9 & 60 & 1211,52169 \\
\hline 0,9 & 50 & 1009,60141 \\
\hline 0,9 & 40 & 807,681126 \\
\hline
\end{tabular}

\section{DISCUSION DE LOS RESULTADOS}

Los datos cinéticos calculados experimentalmente se ajustan a los datos obenidos en la bibliografia.

La reacción de hidrólisis de Cristal Violeta es una reacción de seudoprimer orden y la reacción de saponificación del acetato de etilo es una reacción de segundo orden
El volumen para el reactor PFR calculado para la hidrólisis de Cristal Violeta es de $613 \mathrm{~cm}^{3}$ y la longitud es de $9,65 \mathrm{~m}$

El volumen para el reactor PFR calculado para la saponificación es mayor que para la hidrólisis.

El grado de conversión tanto para la hidrólisis de cristal violeta como para la saponifición esta alrededor del $80 \%$. para las especificaciones de diseño del reactor construido.

El reactor PFR construido es un reactor de polietileno de $12 \mathrm{~m}$ de longitud y de $095 \mathrm{~cm}$ de diámetro interno. El PFR está sumergido en un recipiente de acrílico de $5 \mathrm{~mm}$ de espesor, de $30 \mathrm{~cm}$ de altura y $15 \mathrm{~cm}$ de diámetro interno que se encuentra.

El recipiente de acrílico está lleno de agua termostatizada con la finalidad de variar la temperatura del sistema a voluntad.

\section{CONCLUSIONES}

1. La construcción del reactor se ha realizado con materiales que se encuentran en el mercado.

2. Como el volumen calculado para el PFR para hidrólisis de Cristal Violeta difiere del volumen calculado para la saponificación de acetato de etilo, se ha visto por conveniente normalizar la altura de $12 \mathrm{~m}$ de longitud para el reactor PFR que se pretende construir, haciendo un volumen de $850 \mathrm{~cm}^{3}$ para obtener un grado de conversiónaproximada del $80 \%$.

3. Se ha construido un equipo para que los estudiantes puedan realizar sus prácticas de laboratorio en el curso de Diseño de Reactores.

4. Se obtuvieron resultados satisfactorios para ambas reacciones, tanto para la hidrólisis del cristal violeta como para la saponificación del acetato de etilo y esto se valida al determinar y comparar las constante especificas de velocidad de reacción para cada caso, asi como de la obtención de un grado de conversión aceptable que oscila alrededor del $90 \%$.

5. Las curvas de calibración obtenidas demuestran ser totalmente confiables, debido a que el nivel de confianza es de la unidad.

6. En el caso de la hidrólisis del cristal violeta, se comprobó satisfactoriamente el valor de la constante especifica de reacción, con el reportado por Cayrol y Hundgins. Lo mismo ocurre ene I caso de la saponificación del acetato de etilo.

7. El volumen del reactor PFR construido es de $650 \mathrm{~cm}^{3}$, un diámetro de $0,9 \mathrm{~cm}$ y una longitud de $10.21 \mathrm{~m}$ que permitirá obtener grados de conversión del $90 \%$ para el proceso de Hidrólisis.

En cambio para e proceso de saponificación del Acetato de Etilo se tendrá que disminuir el caudal para obtener grados de conversión altos. 


\section{BIBLIOGRAFIA}

Folgler,H.Scott, Elementos de Ingenieria de las reacciones químicas. Edit. Prentice Hall. Tercera Edición, México, 2001.

Levenspiel Octave, Ingenieria de las reacciones quimicas. Edit Reverte, México, 1993.

Smith, J.M., Ingeniería de la cinética química. Edit. Cesca, México, 1992.

Levenspiel Octave, EL omnilibro de los reactores químicos. Edit Reverte, México,1986.
Levenspiel Octave, El Mininilibro de los Reactores Químicos. Edit Reverte, México 1986.

Luyben, W. A feed-effluent heat exchanger/reactor dynamic control laboratory experiment.(2000) 56-61. Skoog,D.H. Holler,J. Nieman,T. Principios de Análisis Instrumental.Quinta edición. EditorialMc Graw Hill/ Interamericana de España 2001.

Aris R. Análisis de Reactores. Editorial Alambra.Primera Edición en Español. España 1973. 